\title{
Magnetic Water Treatment in Agriculture Sciences
}

\author{
Ammar Subhy Shaker, Faez Saleh Mohammed ${ }^{\text {a) }}$ and Hawraa G.T.Al-Shebani \\ Faculty of Agriculture, University of Al-Qadisiah, Iraq. \\ a) Corresponding Author: faezsalih18@gmail.com
}

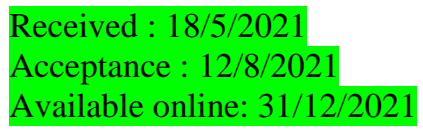

\begin{abstract}
This study investigates the difference in the effect of magnetized water on accelerating the transport of nutrients in sandy soils and on their absorption by citrus fruits [1]. The response of the fruits to irrigation with magnetized water was estimated for three successive seasons. The results showed the effect of magnetized water on the mobility of nutrients in the root zone, which differed significantly from the element to another according to the element's ability to be magnetized. The increase in the extraction of elements from the soil as a result of magnetization was the highest possible for iron, iron extraction reached 9 times the amount of extraction in the normal case from one piece of land. Zinc increased 5 times [2], phosphorous increased 3 times and the increase in manganese was only $80 \%$.In any case, the leaves content of manganese showed the maximum increase of this element and zinc came after it in the second place, while the content of iron in the leaves was the lowest. The leaf content of phosphorous was tripled, and for potassium the increase was significant, while for nitrogen it was not affected by magnetized water. The amount of fruits reached its maximum when the water moved to a distance of 600 meters after the magnetization device on the other hand, the magnetization device stopped causing significant changes on the water that travels for a distance of more than 700 meters [3].
\end{abstract}

Keywords. Magnetic Water, Treatment, Agriculture.

\section{INTRODUCTION}

Agricultural production is an essential component of national income and Arab food security, so it was necessary to work on improving and increasing it, whether through horizontal or vertical expansion, and when horizontal expansion of the cultivated areas was difficult, whether due to desertification that crawls over large areas of agricultural land or as a result of Due to the massive population increase [4], it was necessary to focus on the vertical expansion of agriculture by using genetically improved varieties or by following modern scientific techniques used in other agricultural operations, whether irrigation, fertilization, service operations, etc. Given the great importance that modern technologies can play in improving the reality of Arab agricultural work and in Increasing the productivity of the unit area and thus achieving the so-called vertical expansion in agriculture. Defining these techniques and working to bring them into use must be a priority for any agricultural engineer, and from here we deduced the subject of our research to introduce a modern technology used in the field of agriculture, which is the magnetic water treatment technology [2].

\section{MATERIALS AND METHODS}

\section{- Definition of Magnetic Water Treatment}

This technique is considered as the birth of a new science called biomagnetism, which is a modern technique in which devices manufactured specifically for this purpose are used Magnetotron They are of different sizes. These devices create a very intense concentration of the magnetic field through the wall of the tube to reach the water and contribute to its treatment. This very strong and intense magnetic field generated by the device .Magnetotron It works to bring about a change in the properties of water, whether physical or chemical, as it becomes more energy, vitality and more flowing, due to its effect on the hydrogen bonds in liquid water, which are greatly affected by the magnetic and electric field, as the hydrogen bonds are broken and the properties of water adapt and make it more capable of (dissolving ) reducing surface tension [4].

\section{- Factors That Affect The Degree of Magnetization}

There is a relationship between the velocity of water flow, the amount of water to be treated, and the density of the magnetic field, as he determined the factors on which the degree of magnetization depends, namely: 
1. The amount of liquid prepared for magnetization.

2. The strength of the magnet used .

3. The contact time between the liquid and the magnet [5].

\section{- Water}

Water is the liquid of life and constitutes the highest percentage of the chemical components that enter into the composition of the bodies of living organisms, regardless of their many forms and shapes. This percentage ranges between $60-95 \%$ of the total soft weight of different cells and tissues. It is often considered ) wrongly ( an inert liquid, but on the contrary, it is considered a highly effective substance with unique and distinctive characteristics [6].

\section{- The Polar Structure of Water and The Grouping of Its Molecules}

Water is a liquid with a special reaction, as its molecules are formed with groups with special continuous dynamics that work to separate these groups and collect them in other formations [7], and that water cannot be considered chemically pure, but rather is a clear solution that contains inorganic compounds. It is known that water is composed of hydrogen atoms. and oxygen, and that the water molecule is very simple linked with each other by hydrogen bonds and these bonds may be double or multiple, it may reach dozens of bonds where a water molecule can link with four other molecules and this group is linked with another group and the distance between the hydrogen bond determines the state of water whenever it is close ) Strong attraction ( Water is in a solid state, and as the distance increases, its fluidity (increases) the strength of the bond decreases [8].

\section{- $\quad$ The Solubility of Water}

The polar properties of water molecules help to dissolve many substances due to its high ability to surround the charged particles and separate them from each other, so a layer of water is formed around the ions that prevents them from being attracted to each other, which makes water in fact a unique solvent . The solubility of substances varies from one substance to another. There are substances that dissolve in water at a high rate, such as sugar and salt [5] Such substances are usually described as being easy to dissolve in water, and there are some substances and salts whose solubility is not noticeable in water such as calcium carbonate and barium sulfate, so they are described as useless substances. Solubility [6].

\section{- Water and Magnetism}

The magnetic treatment of water does not actually magnetize water, as is commonly mistaken. Water is not like magnetized materials when exposed to a strong magnetic field [7]. However, as is the case with all liquids, it possesses the properties of magnetic materials. When exposed to a magnetic field, water will produce a weak magnetic field in the direction On the contrary, modified or conditioned water or magnetically treated water is the correct expression to avoid confusion. The magnetic treatment of water does not actually magnetize water, as is commonly mistaken. Water is not like materials that can be magnetized when exposed to a strong magnetic field, except that, as is the case with all liquids, it has properties Materials are magnetic. When exposed to a magnetic field, water will produce a weak magnetic field in the opposite direction. Therefore, modified or air-conditioned water or magnetically treated water is the correct expression to avoid interference [8].

\section{- $\quad$ The Effect of Magnetic Field on Molecules}

The molecules of substances are of two types, they are either polar, such as water and $\mathrm{N}-2 \mathrm{o}$ or non-polar like $\mathrm{O}$ $2, \mathrm{H} 2$.The passage of non-polar particles through the magnetic field will lead to their charge and transform them into a bipolarity [9].

\section{- Effects of Magnetism on The Molecular Structure of Water}

Some studies dealt with the effect of magnetism on the molecular structure of water that placing water molecules within a magnetic field leads to a change or disintegration of hydrogen bonds, and this disintegration works to absorb energy and reduces the level of the union of molecules [6]. 


\section{- Devices Magnetron Features and Installation Methods}

Magnetically treated water can be prepared by devices specially manufactured for this purpose called Magnetotron They are of different sizes that can be installed on the pipes and start from the diameter0.25-30 ENG, which ensures its use for small ponds in gardens to large areas, for example, the device with a diameter 6 Enough to cover a planted area of up to 25 hectares [11].

1. Most of the magnetizing devices are designed in a way that allows them to be tightened firmly and tightly on the irrigation pipes.

2. These devices are separated into two halves and tightened tightly around the tubes, where the screws are tightened with a wrench.

3. It is suitable for all types of tubes, even those that do not carry magnetic current, such as polyvinyl chloride tubes, copper tubes, stainless steel tubes, and rubber tubes.

4. They are installed on the outside of the pipes where they are not affected by the external weather and can be buried in the soil if we take into account the possibility of theft.

5. You do not need a power source and do not need to connect an electric wire to the soil.

6. It is not possible to install it on steel pipes or galvanized any effect on it with electric current.

7. It must be installed at least 1 meter away from any 3-phase electric motor.

8. They must be installed before the first exit to the water, in order to obtain the greatest possible benefit.

9. They should be installed after any water storage in tanks because water will lose its treatment effects if stored for any period of time.

10. It should be avoided in a place where it is exposed to a temperature of more than 70 degrees [10].

\section{- Benefits of Magnetically Treating Water}

Magnetized water has many benefits, the most important of which are:

1. The magnetization of the water prevents the formation of lime deposits on the inner surfaces of the irrigation pipes, which leads to a decrease in the inner diameter of the pipe, which leads to inefficiency, blockage, in addition to the complete destruction of the pipe or device. This feature is considered one of the most important benefits of magnetized water, and the following pictures illustrate this problem .

2. Studies have proven the possibility of using salt water in irrigation by passing it through a magnetic field, as this leads to the dismantling of saline compounds and their analysis into their ions, which removes their harmful effect on the plant, and the use of magnetic technology in the treatment of irrigation water improves soil conditions and reduces salinity By increasing the speed of salt washing and ridding the root area of its damage.

3. As for fertilizers, their obstacles arise through the excessive use of them, and their high prices have made this cumbersome for farmers, in addition to their overlap with many factors that may affect the readiness of the optimal elements for absorption. For absorption with the ease of absorption of water and nutrients dissolved in it, which was eventually reflected in an increase in the speed of growth and the outcome while reducing costs.

4. The loss of vitality of the seeds made it important to raise their vitality, especially the seeds of rare plants, in order to increase the rate and speed of their germination, and the formation of a solid surface layer, especially in limestone soils, may prevent them from germinating. The possible treatment through magnetic technology where emerged in recent years technology as an effective means in the vitality of raising the seed and increase the percentage of germination in experiments using the technique of magnetic seeds and irrigation water obtained an increase in the productivity of wheat, sesame, maize and rates 24,33 and \%24 on It also obtained an increase in the percentage of germination of wheat and tomato seeds by 20 and , \%65 respectively [12].

\section{- Some Practical Examples}

\section{First example:}

A study was conducted on the effect of a stable electromagnetic field on the root growth of radish seeds and its effect on the number of root hairs growing on these roots. The results showed that there was no effect of this field along the roots, but there was Significant increase in the number of root hairs formed on these roots [13]. 


\section{Second example:}

The effect of magnetized water on seed growth was studied. The consistent groups of seeds ) with an average of 10 seeds in each group ( were treated with different types of water) magnetized and non-magnetized water .( In order to know the effect of the magnetic field on the water in addition to its effect on the seeds $[14,15]$.

\section{- $\quad$ Experience}

Two groups of chickpea seeds were selected so that each group contains 10 seeds. The selection criteria depend on their size, and weight ) they almost have the same size and weight, ( and they have a light yellow color and have a surface free of impurities. Each seed is planted and sown in a plastic dish at a depth of $3 \mathrm{~cm}$ within Soil group (1) was watered daily with magnetized water. On the other hand, the second group was irrigated with ordinary water from the tap. The magnetic water is prepared using a magnet that generates a permanent field $(0.23)$ which is placed outside the container in which the water is located, in the form of an opposite reed or against each seed that was watered daily with about $20 \mathrm{ml}$ per day after about four days. Plants begin to grow and appear above the surface of the soil. After the fourth day, plant growth is measured daily [16].

\section{RESULTS AND DISCUSSION}

$\rightarrow$ The hummus from the first group and three of the second group did not grow and did not give any results.

$\rightarrow$ The eight plants that remained from the first group and seven plants that survived from the second group were considered samples under the test [17].

$\rightarrow$ The data was collected over a period of 18 days.

$\rightarrow$ The average value of the length of the tested plants and controlled began to differ between the third and fourth day.

$\rightarrow$ The final value of the length was obtained on the $18^{\text {th }}$ day.It was found that the mean length of the tested group was about $2.67 \mathrm{~cm}$ more than the mean length of the control group .

$\rightarrow$ The magnetic field affects the properties of water. It was found that the value of the acid number of water changes from 7 to 7.6 and the conductivity changes to 4.29 The surface tension of water decreases by an average of.$- \% 2$ Based on these results, it is clear that the magnetic field significantly affects the water and therefore affects the plant is known that trees and plants need many minerals and microelements found in the soil in order for these plants to carry out their functions and also to carry out the process of photosynthesis in all cases. into the soil and becomes absorbable by the plant Also, the consumption of these nutrients from the soil is little and very rare when plants begin to grow and require larger amounts of nutrients. The deficit in nutrients and microelements in the soil is the main reason for low growth rates and low yields of crops. In addition, when the plant is watered using hard and non-magnetic water, a white layer is formed. On the surface of the soil, this is calcium bicarbonate, bicarbonate and some sodium bicarbonate washed away by water penetrating the soil and preserved in the plant roots. The plants then begin to suffocate because of this accumulation in the roots. The results of this process are to reduce the natural growth of plants anyway. Plants that are irrigated using water treated with magnetized water, Easily take mineral salts from the soil without forming any sedimentation on the surface of the soil and the consequential results are an increase in crop productivity and an increase in the quality of agricultural products [18].

\section{REFERENCES}

[1] Davis, RD and WC, Rawls. 1996. Magnetism and its effect on the living system. Environ. Inter, 22(3): 229-232.

[2] Barefoot, RR and C.S. Reich. 1992. The calcium factor: The scientific secret of health and youth. South eastern, PA: Triad Marketing; 5th edition.

[3] Blake, W. 2000. Physical and biological effects of magnet. In : The art of magnetic healing. (Ed. Santwani, MT). B. Jain. Indian Gyan. Com.

[4] EE Fesenko, VI Popov, VV Novikov and SS Khutsyan, Structure formation in water by the action of weak magnetic fields and xenon. Electron microscopic analysis, Biophysics 47 (2002) 365-370.

[5] HEL Madsen, Crystallization of calcium carbonate in magnetic field in ordinary and heavy water, J. Crystal Growth 267 (2004) 251-255

[6] H. Inaba, T. Saitou, K. Tozaki, and H. Hayashi, Effect of the magnetic field on the melting transition of H 2 O and D 2 O measured by a high resolution and supersensitive differential scanning calorimeter, J. Appl. Phys. 96 (2004) 6127-6132. 
[7] Hilal, MH and MM Hilal. 2000a. Application of magnetic technologies in desert agriculture II - Effect of magnetic treatments of irrigation water on salt distribution in olive and citrus fields and induced changes of ionic balance in soil and plant . Egypt. J. Soil. Sci, 40 (3): 423 - 435.

[8] Hilal, MH and MM Hilal. 2000b . Application of magnetic technologies in desert agriculture I - seed germination and seedling emergence of some crops in a saline calcareous soil . Egypt. J. Soil. Sci. 40 (3): 413-422.

[9] Ichiro, O. and S. Ozeki. 2006. Does magnetic treatment of water change its properties. J. Phys. Chem, 110(4): 1509-1512. (Abst).

[10] Igor, S. 2003. Activated water. Electronic Journal of Biotechnology ISSN: No: $1: 0717$ 3458. (https://www.tspace.library.utoronto.ca/bitstream/1807/1359/1/ej03016.pdf.).

[11] I. Otsuka and S. Ozeki, Does magnetic treatment of water change its properties? J. Phys. Chem. B 110 (2006) 1509-1512.

[12] Jiles, D. 1992. Introduction to magnetism and magnetic materials. Chapman and Hill , NY

[13] Jiles, D. 1992. Introduction to magnetism and magnetic materials. Chapman and Hill , NY

[14] JSBaker and Simon. J. Judd, Wat., Res.Vol.30, No.2, pp 247-260 (1996).

[15] JT Trevors and GH Pollack, Hydrogel origin of life; Hypothesis: the origin of life in a hydrogel environment, Progr. Biophysics. Mol. Biol. 89 (2005) 1-8.

[16] J. Yang, S. Meng, LF Xu and EG Wang, Ice tesselation on a hydroxylated silica surface, Phys. Rev. Lett . 92 (2004) 146102.

[17] K. Esaki, F. Ninomiya, K. Hisaki, T. Higasa, K. Shibata, K. Murataand S. Aibara, Effects of high voltage electric field treatment on the water activity of bread, Biosci. Biotech. Biochem. 60 (1996) 1444-1449.

[18] White, K.M.; Hyde, M.K.(2011) The role of self-perceptions in the prediction of household recycling behavior in Australia. Enviro. Behav.44, 785-799. 